\title{
The Structure of the Relaxed Thick Filaments from Lethocerus Flight Muscle
}

\author{
Zhongjun $\mathrm{Hu}^{1}$, Dianne W. Taylor ${ }^{1}$, Michael K. Reedy ${ }^{2}$, Robert J. Edwards ${ }^{2}$ and Kenneth A. Taylor ${ }^{1}$ \\ ${ }^{1}$ Florida State University, Institute of Molecular Biophysics, Tallahassee, FL 32306-4380 \\ ${ }^{2}$ Duke University Medical Center, Department of Cell Biology, Durham, NC 27607
}

Flight muscle thick filaments consist of myosin II and several types of non-myosin proteins. Myosin II consists of two heavy chains and two pairs of light chains, an essential light chain (ELC) and a regulatory light chain (RLC). The first $\sim 850$ residues make up the head which possesses the catalytic and actin binding activity and the remaining $\sim 1100$ residues forming a long $\alpha$-helical coiled-coil, which is essential for thick filament formation. Non-myosin proteins likely to be visible in the flight muscle thick filament reconstruction are paramyosin [1], flightin [2], myofilin [3]. How these proteins are organized in thick filaments is largely unknown as is the detailed myosin rod structure. Our cryoEM structure of relaxed Lethocerus flight muscle thick filaments at $5.5 \AA$ resolution shows the myosin rod in unprecedented detail and suggests the arrangement of at least three non-myosin proteins.

Images were collected automatically using LEGINON [4] on a Titan-Krios equipped with a DE-20 direct electron detector. Each micrograph consisted of 48 frames, total dose $65 \mathrm{e}^{-} / \AA^{2}$, aligned and summed using damage compensated motion correction [5]. A version of RELION 1.2 adapted for iterative helical real space reconstruction [6] was used to process a total of 26,000 filament segments. Micrographs of frozen hydrated relaxed thick filaments show pronounced shelves of density spaced $145 \AA$ apart along the filament axis (Fig. 1). These are generally referred to as "crowns". The rod $\alpha$ helices of a single myosin can be traced in their entirety after generation of a density map consisting of 11 crown repeats (Fig. 2). Myosin rods are localized within a $53 \AA$ wide annulus and run parallel with the filament axis with a slight radial tilt. Inside of the myosin rod annulus is a second annulus containing paramyosin (Fig. 3). Threaded among the myosin rods are four non-myosin densities (Fig. 3A), which may be domains of flightin or myofilin, the only known proteins that are reasonable candidates.

Previous low-resolution relaxed thick filament structures $(<20 \AA)$ from other muscle types revealed an intramolecular interaction between myosin heads [7], dubbed the interacting heads motif (IHM). The myosin heads of Lethocerus thick filaments also form the IHM, but its position is different from other relaxed thick filaments. Instead of lying flat against the filament backbone, the IHM extends perpendicular to the filament surface in a position where it makes no stabilizing contacts with nearest neighbors (Fig. 4). Our results demonstrate the ubiquity of the myosin interacting heads motif in relaxed muscle and show for the first time non-myosin proteins embedded among the myosin rods.

\section{References:}

[1] Bullard B, Luke B, Winkelman L. J Mol Biol 75 (1973), p. 359.

[2] Vigoreaux JO, Saide JD, Valgeirsdottir K, et al. J Cell Biol 121 (1993), p. 587.

[3] Qiu F, Brendel S, Cunha PM, et al. J Cell Sci 118 (2005), p. 1527.

[4] Suloway C, Pulokas J, Fellmann D, et al. J Struct Biol 151 (2005), p. 41.

[5] Wang Z, Hryc CF, Bammes B, et al. Nat Commun 5 (2014), p. 4808.

[6] Clemens DL, Ge P, Lee BY, et al. Cell 160 (2015), p. 940.

[7] Woodhead JL, Zhao FQ, Craig R, et al. Nature 436 (2005), p. 1195. 
[8] The authors acknowledge funding from NIH grants R01 GM030598 and R01 AR014317. ZH was partially supported by a predoctoral fellowship from the American Heart Association.

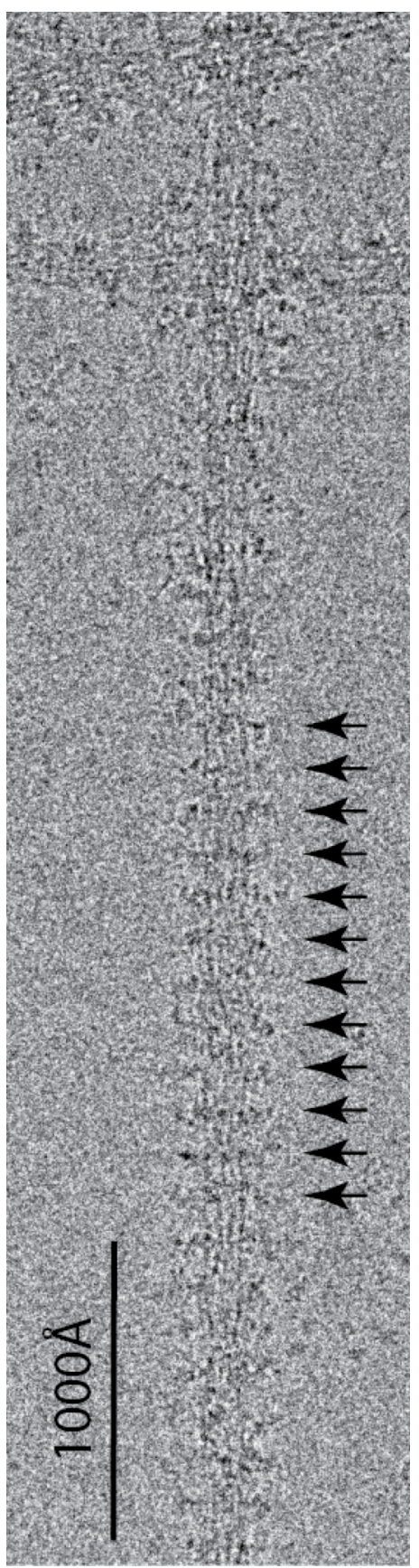

Figure 1. Micrograph of a frozen hydrated Lethocerus indicus flight muscle thick filament. Arrows mark 12 successive crowns, or approximately the length of one myosin molecule.

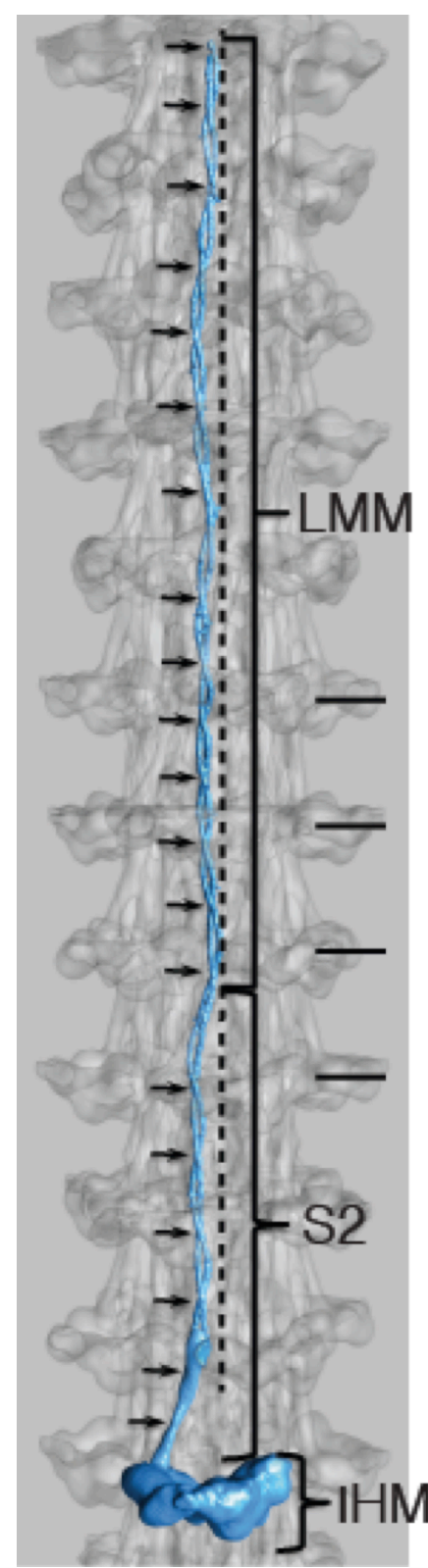

Figure 2. The thick filament reconstruction with a single myosin molecule segmented (light blue). Small arrows mark crossovers in the coiled-coil.
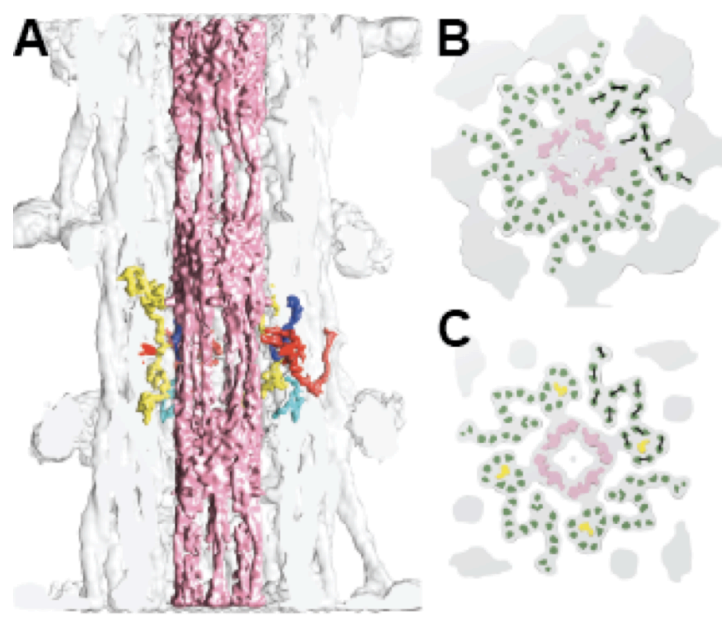

C

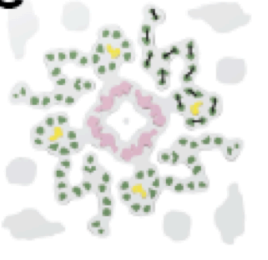

Figure 3. Non-myosin densities. (A) Paramyosin core (salmon) segmented from the reconstruction. Paramyosin has a different symmetry from the myosin and so is poorly represented in the $3 \mathrm{D}$ image. In addition to paramyosin, four additional non-myosin densities appear between the crowns, colored red, yellow, blue and cyan. (B) Transverse section through the reconstruction at approximately the level of the crowns. Each of the green densities represents a single $\alpha$-helix. Paramyosin appears as four paired densities (salmon). (C) A transverse section near the region of greatest paramyosin density.

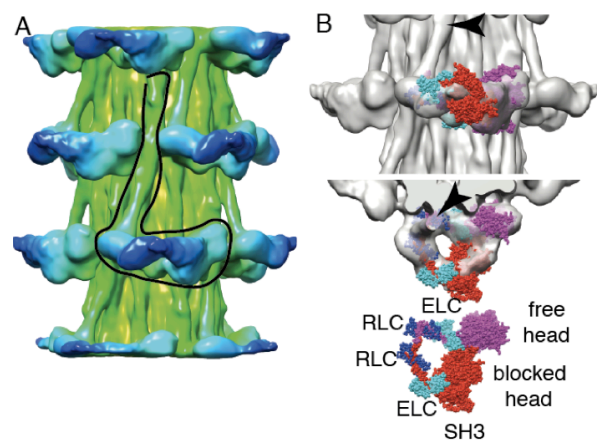

Figure 4. Interacting Heads Motif. (A) Surface view of the reconstruction with radial color coding. Blue densities are at highest radius. (B) With IHM atomic model. Top is a longitudinal view, middle is a transverse view and bottom is the atomic model alone. 